\title{
MODULARITY OF A FAMILY OF ELLIPTIC CURVES
}

\author{
Fred Diamond and Kenneth Kramer
}

We shall explain how the following is a corollary of results of Wiles [W]:

Theorem. Suppose that $E$ is an elliptic curve over $\mathbf{Q}$ all of whose 2division points are rational, i.e., an elliptic curve defined by

$$
y^{2}=(x-a)(x-b)(x-c)
$$

for some distinct rational numbers $a, b$ and $c$. Then $E$ is modular.

Recall that Wiles proves that if $E$ is a semistable elliptic curve over $\mathbf{Q}$, then $E$ is modular [W, Thm. 0.4]. He begins by considering the Galois representations $\bar{\rho}_{E, 3}$ (respectively, $\rho_{E, 3}$ ) on the 3 -division points (respectively, 3-adic Tate module) of $E$. If $\bar{\rho}_{E, 3}$ is irreducible, then a theorem of Langlands and Tunnell is used to show that $\bar{\rho}_{E, 3}$ arises from a modular form. Wiles deduces that $\rho_{E, 3}$ also arises from a modular form by appealing to his results in [W, Ch. 3] and those with Taylor in [TW] to identify certain universal deformation rings as Hecke algebras. This suffices to prove that $E$ is modular if $\bar{\rho}_{E, 3}$ is irreducible. When $\bar{\rho}_{E, 3}$ is reducible, Wiles gives an argument which allows one to use $\rho_{E, 5}$ instead.

In fact, Wiles' results apply to a larger class of elliptic curves than those which are semistable [W, Thm. 0.3], and this was subsequently extended in [Di] to include all elliptic curves with semistable reduction at 3 and 5. Rubin and Silverberg noted that an elliptic curve as in the above theorem necessarily has a twist which is semistable outside 2 , and hence, is modular by [Di, Thm. 1.2]. The purpose of this note is to explain how, by a refinement of their observation, the above theorem follows directly from Wiles' work, without appealing to [Di].

Lemma 1 (Rubin-Silverberg). By at most a quadratic twist, an elliptic curve as in the theorem may be brought to the form

$$
E: y^{2}=x(x-A)(x+B)
$$

for some nonzero integers $A$ and $B$ with $A$ and $B$ relatively prime, $B$ even and $A \equiv-1 \bmod 4$. Let $C=A+B$. For odd primes $p$, the curve $E$ has

Received March 24, 1995. 
good reduction at $p$ if $p$ is prime to $A B C$ and multiplicative reduction at $p$ otherwise.

Proof. Note that a curve as in the theorem is isomorphic to one defined by equation (1) for some integers $A$ and $B$ with $A B(A+B) \neq 0$. Let $D=\operatorname{gcd}(A, B)$. Twisting by $\mathbf{Q}(\sqrt{D})$, we may assume that $A$ and $B$ are relatively prime. By translating $x$ or exchanging $A$ and $B$, we may assume that $B$ is even. Finally, if $A \equiv 1 \bmod 4$, we twist again by $\mathbf{Q}(i)$.

The reduction type of $E$ for odd primes $p$ may be determined as in [Se2, $\S 4]$ and [Si1, Ch. VII].

See $[\mathrm{O}, \S \mathrm{I} .1]$ for discussion of the reduction type and conductor of curves given by equation (1), but under certain restrictions in the case $p=2$. See also [Da, Lemma 2.1] for a related case. We treat the reduction type at $p=2$ in the following lemma.

Lemma 2. Suppose that $E$ is an elliptic curve over $\mathbf{Q}_{2}$ defined by the model (1), with $A \equiv-1 \bmod 4$ and $B$ even. The reduction type, conductor exponent $\mathbf{f}_{2}(E)$ and valuation of the minimal discriminant of $E$ are given by the following table:

\begin{tabular}{|c|c|c|c|c|c|}
\hline $\operatorname{ord}_{2}($ B) & 1 & 2 & 3 & 4 & $\nu \geq 5$ \\
\hline Kodaira Symbol & $I I I$ & $I_{1}^{*}$ & $I I I^{*}$ & $I_{0}$ & $I_{2 \nu-8}$ \\
\hline $\mathbf{f}_{2}(E)$ & 5 & 3 & 3 & 0 & 1 \\
\hline $\operatorname{ord}_{2}\left(\Delta_{\text {min }}\right)$ & 6 & 8 & 10 & 0 & $2 \nu-8$ \\
\hline
\end{tabular}

Proof. A twist of $E$ by the unramified extension $\mathbf{Q}_{2}(\sqrt{-A})$ affects neither reduction type nor conductor exponent, and provides a model of the form

$$
y^{2}=x(x+1)(x+s)
$$

with $\operatorname{ord}_{2}(s)=\operatorname{ord}_{2}(B) \geq 1$ and discriminant $\Delta=16 s^{2}(1-s)^{2}$. For the convenience of the reader, we indicate the appropriate translations of model, depending on $\operatorname{ord}_{2}(s)$, so that the explicit criteria of Tate's algorithm [T] may be used.

If $\operatorname{ord}_{2}(s)=1$, then $\operatorname{ord}_{2}(\Delta)=6$. Put $y+x$ for $y$ in (2) to get

$$
y^{2}+2 x y=x^{3}+s x^{2}+s x .
$$

If $\operatorname{ord}_{2}(s)=2$, then $\operatorname{ord}_{2}(\Delta)=8$. Put $x+2$ for $x$ in (3), to get

$$
y^{2}+2 x y+4 y=x^{3}+(s+6) x^{2}+(5 s+12) x+(6 s+8) .
$$

If $\operatorname{ord}_{2}(s)=3$, use the model (3) with $\operatorname{ord}_{2}(\Delta)=10$. If $\operatorname{ord}_{2}(s) \geq 4$, the model (3) is not minimal and may be reduced to

$$
y^{2}+x y=x^{3}+\frac{s}{4} x^{2}+\frac{s}{16} x
$$


with discriminant $s^{2}(1-s)^{2} / 256$. Thus, (4) has good reduction if $\operatorname{ord}_{2}(s)=$ 4 and multiplicative reduction if $\operatorname{ord}_{2}(s) \geq 5$.

To show that an elliptic curve over $\mathbf{Q}$ is modular, we may replace it with one to which it is isomorphic over $\mathbf{Q}$. We may therefore assume that $E$ is defined by equation (1) with $A$ and $B$ as in Lemma 1 . If $E$ has good or multiplicative reduction at $p=2$, then $E$ is semistable and we can conclude from [W, Thm. 0.4] that $E$ is modular. In view of Lemma 2, we may therefore also assume, henceforth, that $\operatorname{ord}_{2}(B)=1,2$ or 3 .

Let $\ell$ be an odd prime. Choose a basis for $E[\ell]$, the kernel of multiplication by $\ell$ on $E$, and let $\bar{\rho}_{E, \ell}$ denote the representation

$$
G_{\mathbf{Q}} \rightarrow \mathrm{GL}_{2}\left(\mathbf{F}_{\ell}\right)
$$

defined by the action of $G_{\mathbf{Q}}=\operatorname{Gal}(\overline{\mathbf{Q}} / \mathbf{Q})$ on $E[\ell]$. For each prime $p$, we fix an embedding $\overline{\mathbf{Q}} \hookrightarrow \overline{\mathbf{Q}}_{p}$ and regard $G_{p}=\operatorname{Gal}\left(\overline{\mathbf{Q}}_{p} / \mathbf{Q}_{p}\right)$ as a decomposition subgroup of $G_{\mathbf{Q}}$ at a place over $p$. Thus, $\bar{\rho}_{E, \ell} \mid G_{p}$ is equivalent to the representation of $G_{p}$ defined by its action on $E[\ell]\left(\overline{\mathbf{Q}}_{p}\right)$. Let $I_{p} \subset G_{p}$ denote the inertia group.

Recall the special role played by the prime $\ell=3$ in Wiles' approach. We simply write $\rho$ for $\bar{\rho}_{E, 3}$. If $\rho$ is irreducible, then $\rho$ is modular by the theorem of Langlands and Tunnell (see [W, Ch. 5]). Since $E$ has good or multiplicative reduction at 3 , we need only verify certain hypotheses on $\rho$ in order to apply [W, Thm. 0.3] to conclude that $E$ is modular. We shall see that if $E$ has additive reduction at $p=2$, then those hypotheses are satisfied, the crucial point being the verification of a local condition at $p=2$. The irreducibility of $\rho$ in this case is a byproduct of our verification. In fact, we have the following stronger result:

Lemma 3. If $\operatorname{ord}_{2}(B)=1,2$ or 3 and $\ell$ is an odd prime, then $\bar{\rho}_{E, \ell} \mid I_{2}$ is absolutely irreducible.

Proof. For the moment, consider the more general case of a representation $\psi: I \rightarrow \mathrm{SL}_{2}\left(\overline{\mathbf{F}}_{\ell}\right)$, where $I$ is the inertia group of a finite Galois extension of $p$-adic fields and $\ell \neq p$ is a prime. Let $\mathbf{b}(\psi)$ denote the wild conductor exponent [Se2, §4.9]. If $\mathbf{b}(\psi)$ is odd, then $\psi$ is irreducible. Indeed, were $\psi$ to be reducible, it would be equivalent to a representation of the form

$$
\left(\begin{array}{cc}
\chi & * \\
0 & \chi^{-1}
\end{array}\right)
$$

But then, because $\mathbf{b}$ is integer-valued and additive on short exact sequences, $\mathbf{b}(\psi)=2 \mathbf{b}(\chi)$ would be even. 
Under the hypotheses of this lemma, the elliptic curve $E$ has additive reduction at 2 and odd conductor exponent $\mathbf{f}_{2}(E)=2+\mathbf{b}\left(\bar{\rho}_{E, \ell} \mid I_{2}\right)$, independent of the choice of odd prime $\ell$. Since $\operatorname{det} \bar{\rho}_{E, \ell} \mid G_{2}$ is an unramified character associated to $\mathbf{Q}_{2}\left(\boldsymbol{\mu}_{\ell}\right)$, the image of $I_{2}$ under $\bar{\rho}_{E, \ell}$ is contained in $\mathrm{SL}_{2}\left(\mathbf{F}_{\ell}\right)$. It follows that $\bar{\rho}_{E, \ell} \mid I_{2}$ is absolutely irreducible.

Remark. When Lemma 3 applies, an analysis of the group structure of $\mathrm{SL}_{2}\left(\mathbf{F}_{3}\right)$ shows that the image of wild ramification at $p=2$ under $\rho$, and hence, $\bar{\rho}_{E, \ell}$, for any odd $\ell$, is isomorphic to the quaternion group of order 8.

Under the hypotheses of Lemma 3, we see that even the restriction of $\rho=\bar{\rho}_{E, 3}$ to $\operatorname{Gal}\left(\overline{\mathbf{Q}} / \mathbf{Q}\left(\boldsymbol{\mu}_{3}\right)\right)$ is absolutely irreducible. Using Lemma 3, one can also easily check the local conditions on $\rho$ appearing as hypotheses in [W, Thm. 0.3]. Since it is left to the reader of [W] to verify that those local conditions are sufficient to apply the central result [W, Thm. 3.3], we shall explain directly how this is done in the case with which we are concerned. Again, we consider, more generally, $\bar{\rho}_{E, \ell}$ for odd primes $\ell$.

First recall that $\bar{\rho}_{E, \ell}$ is unramified at $p$ if $p \neq \ell$ is a prime of good reduction, i.e., if $p$ does not divide $\ell A B C$.

Next we recall how the Tate parametrization is used to describe $\bar{\rho}_{E, \ell} \mid G_{p}$ for primes $p$ at which $E$ has multiplicative reduction (see $[\operatorname{Se} 2, \S 2.9]$ ). Let $F$ denote the unramified quadratic extension of $\mathbf{Q}_{p}$ in $\overline{\mathbf{Q}}_{p}$. Then $E$ has split multiplicative reduction over $F$ and the Tate parametrization (see [Si2, §V.3]) provides an isomorphism

$$
\overline{\mathbf{Q}}_{p}^{\times} / q^{\mathbf{Z}} \cong E\left(\overline{\mathbf{Q}}_{p}\right)
$$

of $\operatorname{Gal}\left(\overline{\mathbf{Q}}_{p} / F\right)$-modules for some $q \in \mathbf{Q}_{p}$ with $\operatorname{ord}_{p}(q)>0$. From this it follows that for each prime $\ell$, there is a filtration of $\operatorname{Gal}\left(\overline{\mathbf{Q}}_{p} / F\right)$-modules

$$
0 \rightarrow \mathbf{Z}_{\ell}(1) \rightarrow T_{\ell}(E) \rightarrow \mathbf{Z}_{\ell} \rightarrow 0
$$

where $T_{\ell}(E)$ is the $\ell$-adic Tate module and $\mathbf{Z}_{\ell}(1)=\lim _{\leftarrow} \boldsymbol{\mu}_{\ell^{n}}\left(\overline{\mathbf{Q}}_{p}\right)$. One then checks that the representation of $G_{p}$ on $T_{\ell}(E)$ is equivalent to one of the form

$$
\chi \otimes\left(\begin{array}{cc}
\epsilon & * \\
0 & 1
\end{array}\right)
$$

where $\chi$ is either trivial or the unramified quadratic character of $G_{p}$ and $\epsilon$ is the cyclotomic character given by the action of $G_{p}$ on $\mathbf{Z}_{\ell}(1)$. It follows that the representation of $G_{p}$ on $E[\ell]$ is of this form as well, but with $\epsilon$ now defined by the action of $G_{p}$ on $\boldsymbol{\mu}_{\ell}$.

Suppose now that $p \neq \ell$ is an odd prime dividing $A B C$. Then the above analysis of multiplicative reduction applies to $\bar{\rho}_{E, \ell} \mid G_{p}$ and shows that $\bar{\rho}_{E, \ell}$ is either unramified or type (A) at $p$ in the terminology of [W, 
Ch. 1]. (The first possibility occurs precisely when $\operatorname{ord}_{p}(A B C)$ is divisible by $\ell$; see $[\mathrm{Se} 2, \S 4]$.)

Suppose next that $p=\ell$. If $p$ divides $A B C$, then the above analysis of multiplicative reduction shows that $\bar{\rho}_{E, \ell} \mid G_{p}$ is ordinary at $p$ in the terminology of [W, Ch. 1]. If on the other hand $p$ does not divide $A B C$, then the elliptic curve $E$ has good reduction at $p$. In fact, the equation (1) defines an elliptic curve $\mathcal{E}$ over $\mathbf{Z}_{p}$ such that $\mathcal{E}_{\mathbf{Q}_{p}}$ is isomorphic to $E_{\mathbf{Q}_{p}}$ (see [Si2, §IV.5]). The kernel of multiplication by $\ell$ on $\mathcal{E}$ is a finite flat group scheme $\mathcal{E}[\ell]$ over $\mathbf{Z}_{p}$. The representation $\bar{\rho}_{E, \ell} \mid G_{p}$ is given by the action of $G_{p}$ on $E[\ell]\left(\overline{\mathbf{Q}}_{p}\right)$, which we may identify with $\mathcal{E}[\ell]\left(\overline{\mathbf{Q}}_{p}\right)$. In this sense, $\bar{\rho}_{E, \ell} \mid G_{p}$ arises from a finite flat group scheme over $\mathbf{Z}_{p}$. Now $\bar{\rho}_{E, \ell} \mid G_{p}$ is reducible if and only if $E$ has ordinary reduction at $p$, i.e., if and only if $\mathcal{E}_{\mathbf{F}_{p}}$ is ordinary. In that case $\bar{\rho}_{E, \ell}$ is ordinary at $p$ in the sense of [W]. On the other hand, $\bar{\rho}_{E, \ell} \mid G_{p}$ is irreducible if and only if $\mathcal{E}_{\mathbf{F}_{p}}$ is supersingular, in which case $\bar{\rho}_{E, \ell}$ is flat at $p$ in the sense of [W, Ch. 1].

Finally, suppose that $p=2$ and $E$ has additive reduction at 2 . Then $\operatorname{ord}_{2}(B)=1,2$ or 3 , and $\bar{\rho}_{E, \ell} \mid I_{2}$ is absolutely irreducible by Lemma 3 . We claim that $\bar{\rho}_{E, \ell} \mid G_{2}$ is of type (C) at 2 in the terminology of Wiles [W, Ch. 1]. Recall that this means that $H^{1}\left(G_{2}, W\right)=0$, where $W$ is the $G_{2^{-}}$ module of endomorphisms of $E[\ell]\left(\overline{\mathbf{Q}}_{2}\right)$ of trace zero. From the triviality of the local Euler characteristic ([Se1, Thm. II.5]), we have

$$
\# H^{1}\left(G_{2}, W\right)=\# H^{0}\left(G_{2}, W\right) \cdot \# H^{2}\left(G_{2}, W\right) .
$$

By local Tate duality ([Se1, Thm. II.1]), we have

$$
\# H^{2}\left(G_{2}, W\right)=\# H^{0}\left(G_{2}, W^{*}\right)
$$

where $W^{*}=\operatorname{Hom}\left(W, \boldsymbol{\mu}_{\ell}\right)$. Therefore, we wish to prove that $H^{0}\left(G_{2}, W\right)$ and $H^{0}\left(G_{2}, W^{*}\right)$ both vanish. But in fact $H^{0}\left(I_{2}, W\right)$ and $H^{0}\left(I_{2}, W^{*}\right)$ already vanish. Indeed, $I_{2}$ acts trivially on $\boldsymbol{\mu}_{\ell}$, from which we deduce that there is a (noncanonical) isomorphism $W^{*} \cong W$ of $I_{2}$-modules; hence, it suffices to show that $H^{0}\left(I_{2}, W\right)=0$. Since $I_{2}$ acts absolutely irreducibly on $\overline{\mathbf{F}}_{\ell}^{2}$, Schur's lemma implies that the only $I_{2}$-invariant endomorphisms of $\overline{\mathbf{F}}_{\ell}^{2}$ are scalars. But the only scalar in $W$ is zero.

Specializing to the case $\ell=3$, we now conclude that the representation $\rho_{E, 3}$ of $G_{\mathbf{Q}}$ on $T_{3}(E)$ arises from a modular form. Indeed, Wiles [W, Thm. 3.3] establishes an isomorphism between the universal deformation ring of type $\mathcal{D}$ and the Hecke algebra $\mathbf{T}_{\mathcal{D}}$, where $\mathcal{D}=\left(\cdot, \Sigma, \mathbf{Z}_{3}, \emptyset\right)$ with

- - as flat or Selmer according to whether or not $E$ has supersingular reduction at 3 ;

- $\Sigma$ as the set of primes dividing $3 A B C$. 
Since $\rho_{E, 3}$ defines a deformation of $\rho$ of type $\mathcal{D}$, the universal property of the deformation ring thus provides a homomorphism $\mathbf{T}_{\mathcal{D}} \rightarrow \mathbf{Z}_{3}$ with the following property: for all $p$ not dividing $3 A B C$, the Hecke operator $T_{p}$ is sent to $a_{p}=p+1-N_{p}$ where $N_{p}$ is the number of $\mathbf{F}_{p}$-points on the reduction of $E \bmod p$.

The definition of $\mathbf{T}_{\mathcal{D}}$ ensures that this homomorphism arises from a normalized eigenform of weight two whose $p^{\text {th }}$ Fourier coefficient is $a_{p}$ for all such $p$. Hence $E$ is modular.

\section{Acknowledgements}

The authors are grateful to Kevin Buzzard, Ken Ribet and Karl Rubin for comments on an earlier version of this note.

\section{References}

[Da] H. Darmon, The equations $x^{n}+y^{n}=z^{2}$ and $x^{n}+y^{n}=z^{3}$, Duke IMRN 10 (1993), 263-274.

[Di] F. Diamond, On deformation rings and Hecke rings, preprint.

[O] J. Oesterlé, Nouvelles approches du "théorème" de Fermat, Séminaire Bourbaki 694 (1987-88), Astérisque 161-162 (1988), 165-186.

[Se1] J. P. Serre, Cohomologie Galoisienne, $5^{e}$ éd, LNM 5. Springer-Verlag, New York, 1994.

[Se2] _ Sur les représentations modulaires de degré 2 de $\mathrm{Gal}(\overline{\mathbf{Q}} / \mathbf{Q})$, Duke Math. J. 54 (1987), 179-230.

[Si1] J. Silverman, The arithmetic of elliptic curves, GTM 106, Springer-Verlag, New York, 1986.

[Si2] _ Advanced topics in the arithmetic of elliptic curves, GTM 151, SpringerVerlag, New York, 1994.

[T] J. T. Tate, Algorithm for determining the type of a singular fiber in an elliptic pencil, in Modular Functions of One Variable IV, LNM 476, Springer-Verlag, New York, 1975.

[TW] R. Taylor and A. Wiles, Ring theoretic properties of certain Hecke algebras, to appear in Ann. Math.

[W] A. Wiles, Modular elliptic curves and Fermat's Last Theorem, to appear in Ann. Math.

D.P.M.M.S., 16 Mill Lane, Univ. of Cambridge, Cambridge, CB2 1SB, UK

E-mail address: f.diamond@pmms.cam.ac.uk

Department of Mathematics, Queens College (CUNY), Flushing, NY 11367

E-mail address: kramer@qcvaxa.acc.qc.edu 